\title{
IS 3D-CT REFORMATION USING FREE SOFTWARE APPLICABLE TO DIAGNOSIS OF BONE CHANGES IN MANDIBULAR CONDYLES?
}

\author{
Marília Gerhardt de OLIVEIRA ${ }^{1}$, Luciano Engelmann MORAIS ${ }^{2}$, Daniela Nascimento SILVA ${ }^{1}$, Helena Willhelm de OLIVEIRA ${ }^{1}$, \\ Cláiton HEITZ ${ }^{1}$, Lêonilson GAIÃO ${ }^{3}$
}

\author{
1- DDS, MSc, PhD, Department of Oral and Maxillofacial Surgery, School of Dentistry, Pontifical Catholic University of Rio Grande do Sul, Porto \\ Alegre, RS, Brazil. \\ 2- DDS, MSc, PhD, Department of Oral and Maxillofacial Surgery, School of Dentistry, PUCRS, Porto Alegre, RS, Brazil. \\ 3- DDS, MSc, PhD student, Department of Oral and Maxillofacial Surgery, School of Dentistry, Pontifical Catholic University of Rio Grande do Sul, \\ Porto Alegre, RS, Brazil.
}

Corresponding address: Prof. Dr. Marília Gerhardt de Oliveira - Av. Cel. Lucas de Oliveira, 1841/203 - Petrópolis - 90460-001, Porto Alegre - RS Brazil -Phone: +55 51 3333-1206 - Fax: +55 51 3330-9545 - e-mail: mogerhardt@yahoo.com.br

Received: April 17, 2008 - Modification: August 31, 2008 - Accepted: October 07, 2008

\begin{abstract}
$O$ bjectives: This study evaluated the agreement of computed tomography (CT) imaging using 3D reformations (3DR) with shaded surface display (SSD) and maximum intensity projection (MIP) in the diagnosis of bone changes in mandibular condyles of patients with rheumatoid arthritis (RA), and compared findings with multiplanar reformation (MPR) images, used as the criterion standard. Material and Methods: Axial CT images of 44 temporomandibular joints (TMJs) of 22 patients with RA were used. Images were recorded in DICOM format and assessed using free software (ImageJ). Each sample had its 3DR-SSD and 3DR-MIP results compared in pairs with the MPR results. Results: Slight agreement $(\mathrm{k}=0.0374)$ was found in almost all comparisons. The level of agreement showed that 3DR-SSD and 3DR-MIP yielded a number of false-negative results that was statistically significant when compared with MPR. Conclusions: 3DR-SSD or 3DR-MIP should only be used as adjuvant techniques to MPR in the diagnosis of bone changes in mandibular condyles.
\end{abstract}

Key words: Helical computed tomography. Image reconstruction. Three-Dimensional Imaging.

\section{INTRODUCTION}

Computed tomography $(\mathrm{CT})$ data can be manipulated in workstations and, more recently, with the use of personal computers (PC) to obtain better interpretations. The standardization of medical images using the Digital Imaging and Communications in Medicine (DICOM) protocol ${ }^{5}$ created an interface between workstations, which generate and export CT data, and PCs, which import and manipulate CT data.

CT data is imported with the use of software packages, some of which are available as free software, such as the ImageJ from the National Institutes of Health. Free software differs from commercial ones in that they are available free of charge. The CT reformation tools of the ImageJ software installed in a PC were used in the present study.

The objective of this study was to evaluate the agreement of CT imaging using 3D reformations (3DR) with shaded surface display (SSD) and maximum intensity projection (MIP) in the diagnosis of bone changes in mandibular condyles of adult patients with rheumatoid arthritis (RA) and compare findings with multiplanar reformation (MPR) images, used as the criterion standard.

\section{MATERIAL AND METHODS}

This study was approved by the Science and Ethics Committee of the School of Dentistry of the Pontifical Catholic University of Rio Grande do Sul, Brazil, and informed consent was obtained from all patients.

The study sample consisted of axial CT images on DICOM format of 44 temporomandibular joints (TMJs) of 22 patients. Patients' ages ranged from 43 to 69 years, 20 were women, and all had RA. All RA diagnoses were made according to the criteria established by the American Rheumatism Association $^{3}$. All patients were at different phases of RA treatment.

All CT images were acquired using a helical CT scanner (CT-Twin, Elscint, Haifa, Israel) at $120 \mathrm{KV}$ peak, $220 \mathrm{~mA}$ and high-resolution mode. The following scanning parameters were used: slice thickness $-1 \mathrm{~mm}$, increment - $0.8 \mathrm{~mm}$, pitch -1.0 ; and field of view $-280 \mathrm{~mm}$. The patient was positioned 
supine on the scanner table, the Frankfurt plane was perpendicular to the ground, and the head was fixed and immobilized by the contention devices of the CT-Twin unit. The number of slices per patient ranged from 31 to 65 (mean $=47.5$ ).

The anatomical region from which images were obtained was on a plane parallel to the Frankfurt plane, started at the orbital floor and extended down to the mandibular notch. This scanning protocol properly recorded all TMJ structures, reduced the chances of artifacts generated by metallic restorations in the lower limit of the acquisition zone, and avoided excessive exposure of orbit structures.

After acquisition, axial CT data were transferred to a PC to evaluate the manipulation of CT images out of a workstation. The ImageJ software was used to segment axial CT data of the mandibular condyles, and sequences of CT planes were obtained by reslicing the volume, which produced the MPR images. From the CT planes, 3DR (SSD and MIP) images were obtained.

The selected views were organized in a separate PowerPoint presentation (Microsoft Corporation, USA) according to the reformation method used: MPR, 3DR-SSD or 3DR-MIP. The reconstructions were randomly assigned to two observers, an experienced radiologist and an oral and maxillofacial surgeon, who were blinded to which group each sample belonged. Analyses were carried out individually and the diagnoses were made by consensus between the two observers. The bone changes investigated were: flattening, osteophytes, erosions, pseudocysts and remodeling (Figures 1-4).

Data were organized regarding the presence or absence of bone changes in each view by each observer to allow

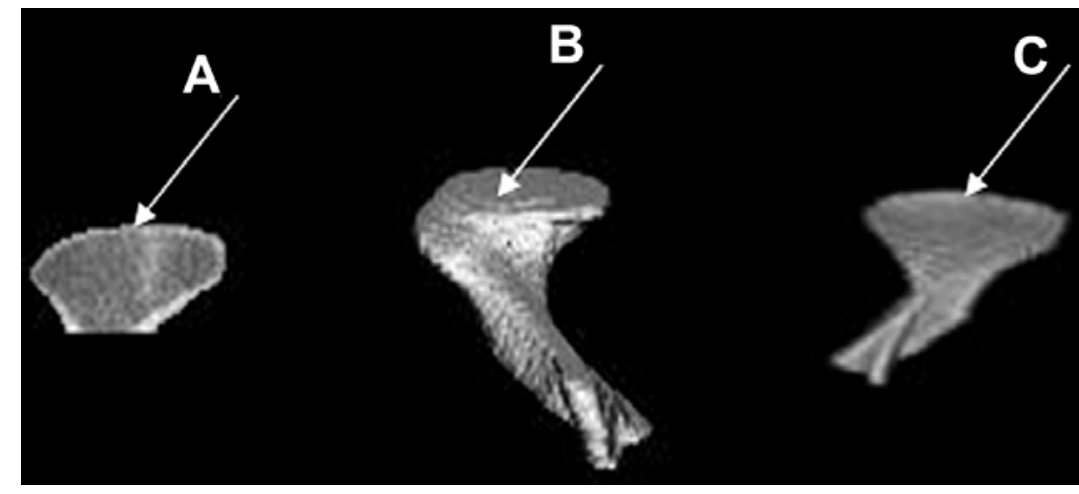

FIGURE 1- MPR (A), 3DR-SSD (B) and 3DR-MIP (C) images showing flattening in the same mandibular condyle

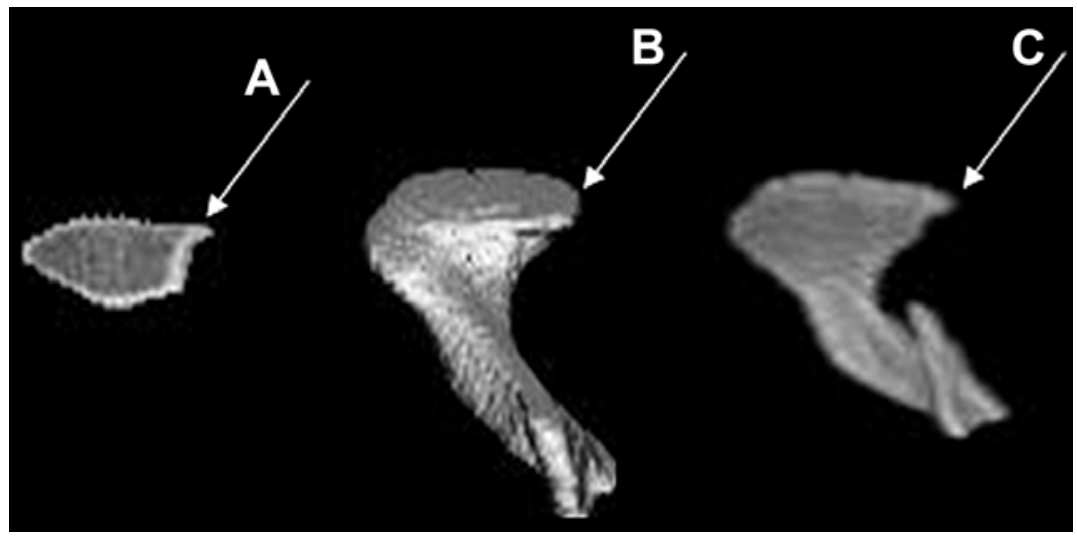

FIGURE 2- MPR (A), 3DR-SSD (B) and 3DR-MIP (C) images showing osteophyte in the same mandibular condyle

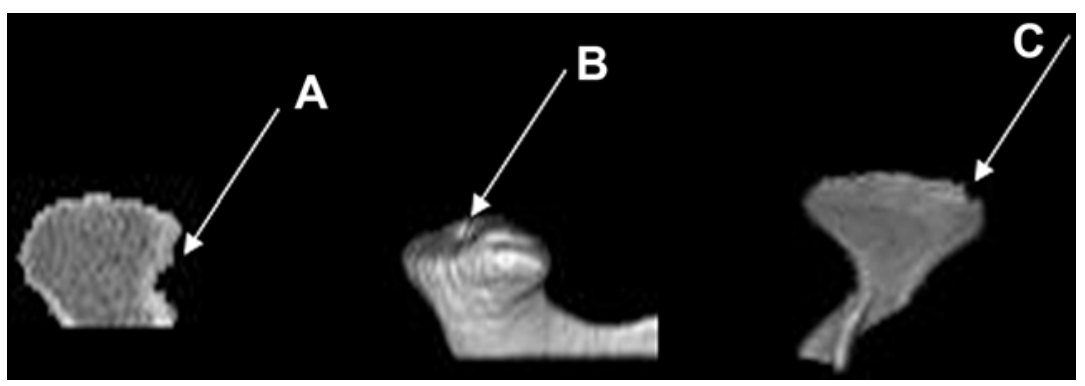

FIGURE 3- MPR (A), 3DR-SSD and 3DR-MIP images showing erosion in the same mandibular condyle 


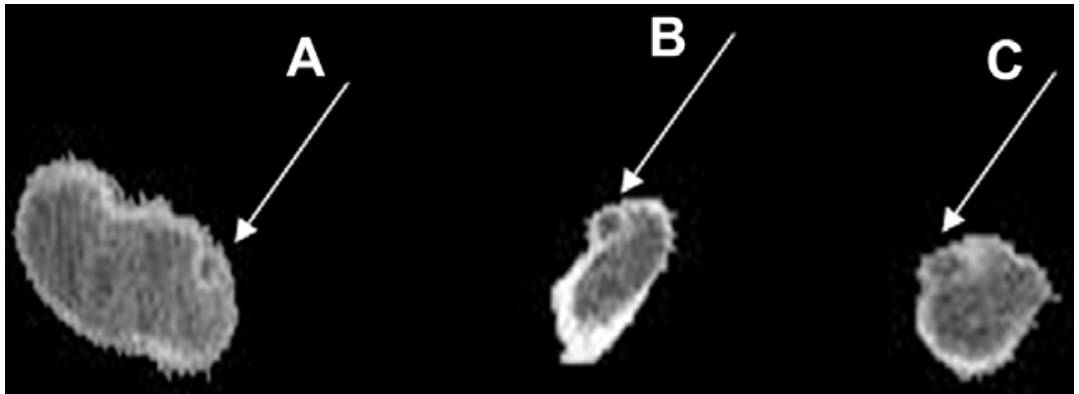

FIGURE 4- MPR images of pseudocysts in mandibular condyles: axial slice (A), sagittal slice (B) and coronal slice (C)

TABLE 1- Relationship between the number of bone changes diagnosed with MPR and with 3DR-SSD, considering each patient individually $(n=22)$

\begin{tabular}{cccc}
\hline Method & Number of bone changes* & $\mathbf{n}^{\dagger}$ & $\mathbf{p}$ \\
\hline \multirow{2}{*}{ SSD - MPR } & SSD < MPR & 15 & 0.003 \\
& SSD > MPR & 2 & \\
SSD $=$ MPR & 5 & \\
\hline
\end{tabular}

$3 D R=3 D$ reformations; 3DR-SSD $=3 \mathrm{D}$ reformations with shaded surface display; MPR = multiplanar reformation; $\mathrm{TMJ}=$ temporomandibular joints.

* < samples with lower number of bone changes diagnosed (false-negative results);

$>$ samples with higher number of bone changes diagnosed (false-positive results);

= samples with same number of bone changes diagnosed.

${ }^{\dagger}$ Number of individuals.

TABLE 2- Relationship between the number of bone changes diagnosed with MPR and 3DR-MIP, considering each patient individually $(n=22)$

\begin{tabular}{|c|c|c|c|}
\hline Method & Number of bone changes* & $\mathbf{n}^{\dagger}$ & $\mathbf{p}$ \\
\hline \multirow[t]{3}{*}{ MIP - MPR } & MIP < MPR & 11 & 0.009 \\
\hline & MIP > MPR & 1 & \\
\hline & MIP = MPR & 10 & \\
\hline
\end{tabular}

$3 D R=3 D$ reformations; 3DR-SSD $=3 \mathrm{D}$ reformations with shaded surface display; MPR = multiplanar reformation; $\mathrm{TMJ}=$ temporomandibular joints.

* < samples with lower number of bone changes diagnosed (false-negative results);

$>$ samples with higher number of bone changes diagnosed (false-positive results);

= samples with same number of bone changes diagnosed.

${ }^{\dagger}$ Number of individuals.

statistical comparison. The Statistical Package for Social Sciences version 14 for Windows (SPSS ${ }^{\mathrm{TM}}$ ) was used for data analysis by a biostatistician. The Wilcoxon test was used for the comparisons of the diagnosis made using MPR (criterion standard) and 3DR-SSD and 3DR-MIP. Kappa statistics was used for the analysis of the agreement between diagnoses of each bone change investigated on axial, sagittal or coronal views by MPR (criterion standard), 3DR-SSD and 3DR-MIP.

\section{RESULTS}

The diagnostic performance of each 3DR method (SSD and MIP) was analyzed in comparison with MPR results for each TMJ to evaluate whether observers diagnosed the same changes found on MPR images, or if 3DR methods yielded significantly more false-negative or false-positive results. Statistically significant differences were found in the comparison of MPR with 3DR-SSD (Table 1) and 3DR-MIP (Table 2), and the 3DR methods yielded significantly more false-negative diagnoses. 
The MPR results for each TMJ underwent paired comparisons with the 3DR-SSD and 3DR-MIP results. Agreement was analyzed using the kappa coefficient. Slight agreement (below 0.374) was found in almost all samples (data not shown).

For the right TMJ, moderate agreement was found between the diagnoses of remodeling in the comparisons of MPR and 3DR-SSD (on the axial view, $\mathrm{k}=0.522$; on the sagittal view, $\mathrm{k}=0.560$ ) and MPR and 3DR-MIP (on the axial view, $\mathrm{k}=$ $0.441)$.

For the left TMJ, moderate agreement was found between the diagnoses of flattening $(\mathrm{k}=0.645)$ and remodeling $(\mathrm{k}=$ 0.450 ) in the comparison of MPR and 3DR-SSD on the coronal view.

\section{DISCUSSION}

The development of CT techniques to acquire, process and manipulate images has improved the knowledge of structures that are otherwise difficult to visualize, such as the TMJ. Data transfer between workstations and PCs, standardization of image formats using protocols such as DICOM, and the use of free software follow a global trend. The manipulation of medical images should be mastered by health professionals that work with diagnosing, planning and treatment of patients because this technology produces additional images to the ones initially reformatted by radiology technicians 5 .

The comparison of the 3DR (SSD and MIP) with the MPR (criterion standard) images of patients with RA is justified because of the high frequency of superficial and subcortical bone changes in the TMJs of these patients ${ }^{2,4,6,7,9}$. The data generated yield a variety of results that can be analyzed statistically. Prevalence of bone findings could be influenced by the course of disease and treatment phase in each patient.

A larger number of bone changes are found in the mandibular condyles than in the mandibular fossa and in the articular tubercle of the temporal bone. The less frequent diagnosis of bone changes in the mandibular fossa of the temporal bone may be explained by the lack of precision of CT imaging of this structure when compared with the mandibular condyles ${ }^{1}$. Because of that, we chose to study segmented mandibular condyles rather than other bone structures of the temporal bone, which compose the TMJ (Figure 1).

The MPR method was chosen as the criterion standard because it provides accurate visualization of superficial and subcortical changes, which are frequently found on the TMJs of patients with RA. The 3DR-SSD method is used worldwide in the examination of TMJs. CT imaging is useful in the evaluation of TMJs of patients with advanced stages of RA ${ }^{7}$, but the results of 3DR-SSD in the analysis of subcortical changes are limited.

The 3DR-MIP method is not frequently used in studies of bone structures ${ }^{8}$. The evaluation of this technique in the examination of TMJs of patients with RA has been proposed because it provides visualization of subcortical changes.
The significant differences found in this study support the hypothesis that the 3DR-SSD and the 3DR-MIP techniques differ from MPR because of their high frequency of falsenegative results.

The false-negative results in diagnosing changes with 3DR (SSD and MIP) may be explained by partial volume effects, distortion of contours of structures that are oblique to the slice, or a slice thickness (wide voxels) that is not adequate to the size of the bone change. Moreover, the 3DR-SSD image formation process is performed in "shading" of the more superficial layer of pixels and, therefore, provides poor visualization of pseudocysts ${ }^{8}$.

When the bone changes of each TMJ were compared separately using both reformation methods (3DR-SSD and 3DR-MIP), the high occurrence of false-negative results for subcortical changes also had an effect on almost all comparisons, for which slight agreement was found.

\section{CONCLUSIONS}

The following conclusions may be drawn: a) 3DR-SSD and 3DR-MIP yielded a number of false-negative results that was statistically significant when compared to MPR; b) 3DRSSD and 3DR-MIP should only be used as adjuvant techniques to MPR in the diagnosis of bone changes in mandibular condyles, according to the levels of agreement found.

\section{REFERENCES}

1- Ahlqvist JB, Isberg AM. Validity of computed tomography in imaging thin walls of the temporal bone. Dentomaxillofac Radiol. 1999;28:13-9.

2- Al-Mobireek AF, Darwazeh AM, Hassanin MB. Experimental induction of rheumatoid arthritis in temporomandibular joint of the guinea pig: a clinical and radiographic study. Dentomaxillofac Radiol. 2000;29:286-90.

3- Arnett FC, Edworthy SM, Bloch DA, McShane DJ, Fries JF, Cooper NS, et al. The American Rheumatism Association 1987 revised criteria for the classification of rheumatoid arthritis. Arthritis Rheum. 1988;31:315-24.

4- Celiker R, Gökçe-Kutsal Y, Eryilmaz M. Temporomandibular joint involvement in rheumatoid arthritis. Relationship with disease activity. Scand J Rheumatology. 1995;24:22-5.

5- Farman AG. Raising standards: digital interoperability and DICOM. Oral Surg Oral Med Oral Pathol Oral Radiol Endod. 2005;99:525-6.

6- Goupille P, Fouquet B, Cotty P, Goga D, Valat JP. Direct coronal computed tomography of the temporomandibular joint in patients with rheumatoid arthritis. Br J Radiol. 1992;65:955-60.

7- Gynther GW, Tronje G, Holmlund AB. Radiographic changes in the temporomandibular joint in patients with generalized osteoarthritis and rheumatoid arthritis. Oral Surg Oral Med Oral Pathol Oral Radiol Endod. 1996;81:613-8.

8- Kawamata A, Ariji Y, Langlais RP. Three-dimensional computed tomography imaging in dentistry. Dent Clin North Am. 2000;44:395-410.

9- Yoshida A, Higuchi Y, Kondo M, Tabata O, Ohishi M. Range of motion of the temporomandibular joint in rheumatoid arthritis: relationship to the severity of disease. Cranio. 1998;16:162-7. 